$\xi=-1$

\title{
Embedded Linux based agricultural field monitoring and automation using IoT
}

\author{
Bhrugumalla L V S Aditya ${ }^{1 *}$, K. Subrahmanyam² \\ ${ }^{I} M$. Tech scholar, Department of Computer Science \& Engineering, \\ Koneru Lakshmaiah Education Foundation, Vaddeswaram, Guntur, PIN: 522502, A.P, India \\ ${ }^{2}$ Professor, Department of Computer Science \& Engineering, \\ Koneru Lakshmaiah Education Foundation, Vaddeswaram, Guntur, PIN: 522502, A.P, India \\ *E-mail: blvsaditya@gmail.com
}

\begin{abstract}
The main idea of this venture is to have an irrigation system which is self-controlled it is also observed that the setup is available for a low price. Wi-Fi technology is used to design this setup. It also aims to reduce manual operation in the agricultural field. The changes taking place are noted. The results obtained are further used to develop the field. As the whole setup is online the readings and values are obtained in real-time a Liquid crystal display is used to know the readings of the conditions.
\end{abstract}

Keywords: Humidity Sensor; LCD; Raspberry-Pi; Soil-Moisture Sensor; Temperature Sensor; Water Level Sensor.

\section{Introduction}

In this project, a low-cost kit is developed so that it can be used in agricultural field at a basic level. Raspberry-pi kit accompanied with wireless Wi-Fi technology is used in this implementation. Effective utilization of limited resources such as water, pesticides etc. is exhibited. Controlling device of entire system is done using raspberry pi. whenever sensor unit gets from respected sensors these inputs are fed to controller ATMEGA 328.it performs appropriate task related to data received like motor ON/OFF control. The live data is continuously uploaded to a predefined Wi-Fi module interfaced to microcontroller and appropriate values are displayed on LCD.

\section{Literature Survey}

- Drip irrigation is controlled by GSM based ZigBee solenoid valve. Real-time support is provided by this technology. This is a control system and monitors each and every activity in the onsite.by this system lot of manpower, power, water is saved. About small percentage of consumption is saved by the use of this prototype.

- Here irrigation control problem is solved by artificial neural network controller. ON/OFF controller is used in this prototype. This is a failed technology. It has its own limitations. A straightforward way to deal with Irrigation control issue utilizing
Artificial Neural Network Controller. The proposed framework is contrasted, and ON/OFF controller and it is demonstrated that ON/OFF Controller based System bombs wretchedly as a result of its impediments. Then again ANN based approach has brought about conceivable usage of better and more productive control. These controllers don't require earlier information of framework and have inborn capacity to adjust to the changing conditions not at all likes customary techniques. It is imperative that ANN based frameworks can spare parcel of resources (energy and water) and can give streamlined outcomes to all sort of farming territories.

- The requirement for frameworks that make agribusiness less demanding and more maintainable includes expanded inside the previous couple of years. The capacity to ration two of the most essential assets of a rancher, water and time, has been the most recent test. A framework that gives this capacity - using proficient and solid techniques, for example, remote sensor organizing, sprinkler water system, GSM, SMS innovations and promptly accessible cell phone gadgets - is sure to enable the ranchers to show signs of improvement yield and on a bigger scale, help the horticultural and monetary development of the nation. 
- There is a developing number of utilization of information mining systems in farming and a developing measure of information that are as of now accessible from numerous assets. This is moderately a novel research field and it is required to develop later on. There is a considerable measure of work to be done on this rising and fascinating examination field. The multidisciplinary approach of coordinating software engineering with agribusiness will help in anticipating/overseeing agrarian products adequately.

- The Automated Wireless Watering System is an easy to understand framework, which advises the client about its status. The 2 methods of operations give the client the choice of programmed and manual process. The framework likewise gives the $\log$ record of the occasions did.

\section{Implementation}

From the above figure, we can see that the gadget which can play out the assignment is a Raspberry Pi processor. There are distinctive sensors, for example, Temperature, Heartbeat, BP. The sensors information is sent to android telephone through Wi-Fi. To play out this assignment, Raspberry Pi processor is customized utilizing installed 'Linux'.

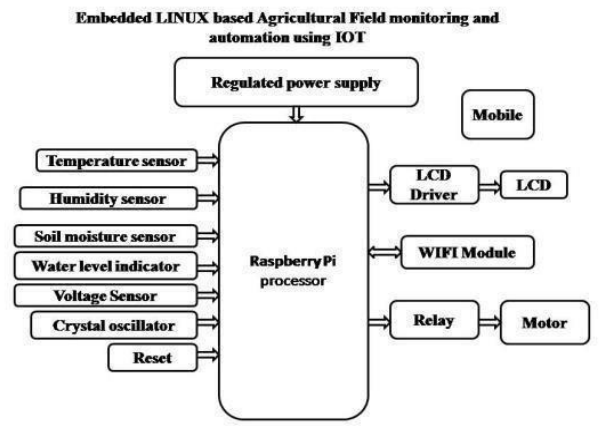

Wi-Fi (Short for Wireless Fidelity) is a remote innovation that utilizations radio recurrence to transmit information through the air.

\subsection{Raspberry Pi processor (ARM-11):}

This framework comprises of ARM-11 small-scale processer which is the primary controlling piece of the framework. The sensor will detect the readings from the human body it can appear in versatile by utilizing WiFi. The concise presentation of various modules utilized as a part of this task is talked about underneath.
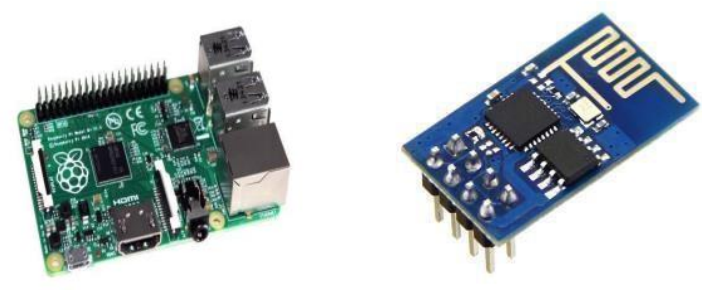

Raspberry pi is a small size computer which was originally invented for the education purpose so that the students can learn more about the hardware and software with low cost. Raspberry pi uses many kinds of processors, which supports several versions of Linux os through which we can surf the internet or play games etc. raspberry pi just looks like a motherboard with many chips, input and output ports to which we can connect many devices like storage devices, computing devices etc. some of the components that the raspberry pi contain are ARM CPU/GPU, GPIO, RCA, LED, USB, HDMI, audio out, SD card slot, ethernet.

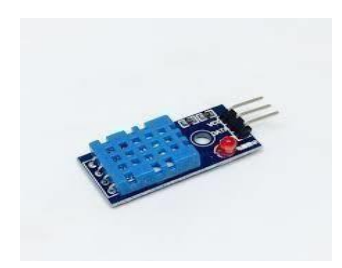

\section{Temperature \& Humidity sensor DHT 11.}

Place the sensor on a breadboard and for the ground connection connect the raspberry pi to the breadboard integrate the code that calculates the humidity of the soil then run the code it will calculate the soil moisture and displays it on the monitor.

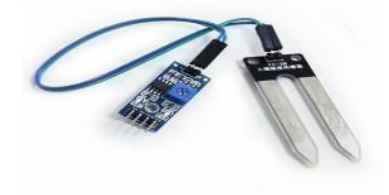

Dampness sensor will be utilized to discover nearness of dampness in the soil. We will embed this sensor into the dirt. It generally checks the nearness of moisture. If the dirt is dry then it will offer data to Microcontroller that the dirt is dry so need to switch on the motor. By this Microcontroller will play out the predetermined activity depending upon our prerequisite. Subsequent to getting adequate dampness the sensor again faculties the nearness of dampness.

\subsection{WIFI Module}

WIFI is the short form of the wireless fidelity.it is a networking technology which uses radio waves to connect many devices like smart phones, computers etc. In order to connect and provide wifi services we need some devices like router, gateways, hubs etc., which will expand the operating range of the wifi. Here in this paper one of the wifi device we used is ESP8266 which is low in cost and uses the tcp/ip protocol.it contains L 10632 bit RISC microprocessor $64 \mathrm{~KB}$ RAM,10 bit ACD,16 GPIO pins.

\subsection{LDR:}

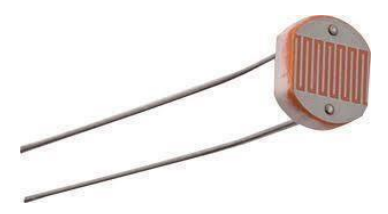


A photograph resistor or light ward resistor is a part that is delicate to light. At the point when light falls upon it then the protection changes. Estimations of the protection of the LDR may change over numerous requests for greatness the estimation of the protection falling as the level of light increments. It isn't extraordinary for the estimations of protection of an LDR or photograph resistor to be a few uber ohms in haziness and afterward to tumble to a couple of hundred ohms in brilliant light. With such a wide variety of protection, LDRs are anything but difficult to utilize.

\subsection{Relay}

This hand-off inside comprises of an acceptance loop and a contact. The loop gets charged when power passes and pulls the contact upwards. We influence utilization of this property of transfer to work the engine. The enlistment loop is associated with the controller and power is permitted to go as for the dampness sensor input. At whatever point the contact of the hand-off is pulled it turns ON the gadget which is associated with the yield of the hand-off.

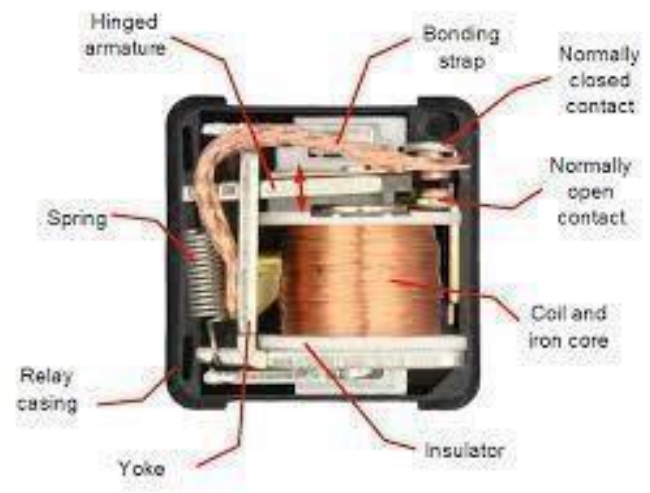

Fig: Inner Picture of Relay

\subsection{LCD:}

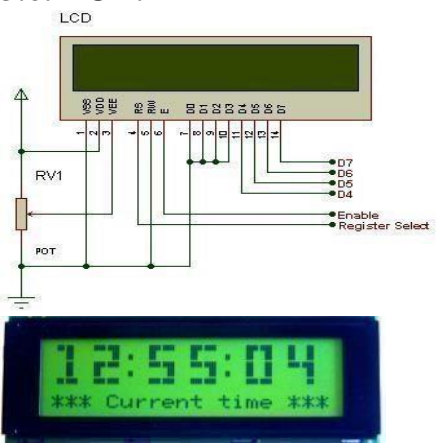

WH2002A is a Character LCD 20x2 show which is worked in with ST7066 controller IC; its default interface is 6800 4/8-bit parallel. These LCD show 20x2 modules are additionally accessible in SPI and I2C interface by utilizing RW1063 controller IC. WH2002A is accessible in a few distinctive backdrop illumination hues including blue, green, white, yellow-green, golden, red and white LEDs. There are diverse character textual styles/writings accessible for ST7066 IC including English/Japanese, English/West European, English/Scandinavian European, or English/Cyrillic (Russian) as alternatives. This module can work at temperature from $-20^{\circ} \mathrm{C}$ to $+70^{\circ} \mathrm{C}$; its stockpiling temperature ranges from $-30^{\circ} \mathrm{C}$ to $+80^{\circ} \mathrm{C}$.

\subsection{Motor:}

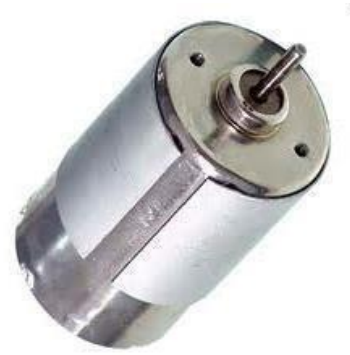

A DC engine is any of a class of rotating electrical machines that believers coordinate current electrical vitality into mechanical vitality. The most widely recognized writes depend on the powers delivered by attractive fields. About a wide range of DC engines have some interior component, either electromechanical or electronic, to intermittently alter the course of current stream in part of the engine.

\section{Development of Project:}

Raspberry pi kit is used for development of project. The python codes written for working of devices are deployed into the kit.

The whole coding is done on Linux environment. temperature sensor, humidity sensor, soil moisture sensor, water level indicator are connected. LCD display is connected to for knowing the values. voltage sensor, motor is also connected to raspberry pi.

\section{Working of Project}

A dedicated AC power supply is provided to the kit. A dedicated internet connection is provided through WIFI. Rectifier converts AC power supply to DC power supply. Different sensors send the values to raspberry pi kit. The values are stored in memory card This system is implemented for agricultural field's environmental monitoring purpose. connected to kit. From the memory card by use of a dedicated IP address one can access the values from the kit.

Difference between previous kit and this kit: Previous kit is costly and now kit is developed in reasonable price. Complexity in the kit is reduced in this project.

\section{Use of the Project for end user:}

The values sent to end user help him to understand more about the agricultural field. Effective usage of pesticides can be established. Water levels in the field can be acquired through which end user can be alarmed. Labor cost for the field can be reduced.

\section{Advantages:}


1. Different parameters monitoring system.

2. Alerts display on LCD.

3. LDR sensor-based sunlight presence detection

4. Humidity detection alerts

5. Visual alerts using LCD

6. Efficient and low-cost design.

7. Lower power consumption.

8. Fast response.

\section{Applications}

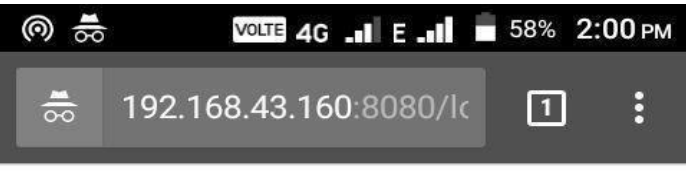

01:59 Temperature: $25.0 \mathrm{C}$

01:59 Humidity: 46.0

01:59 Moisture : perc 0

02:00 Temperature: $25.0 \mathrm{C}$

02:00 Humidity: 46.0

02:00 Moisture : perc 0

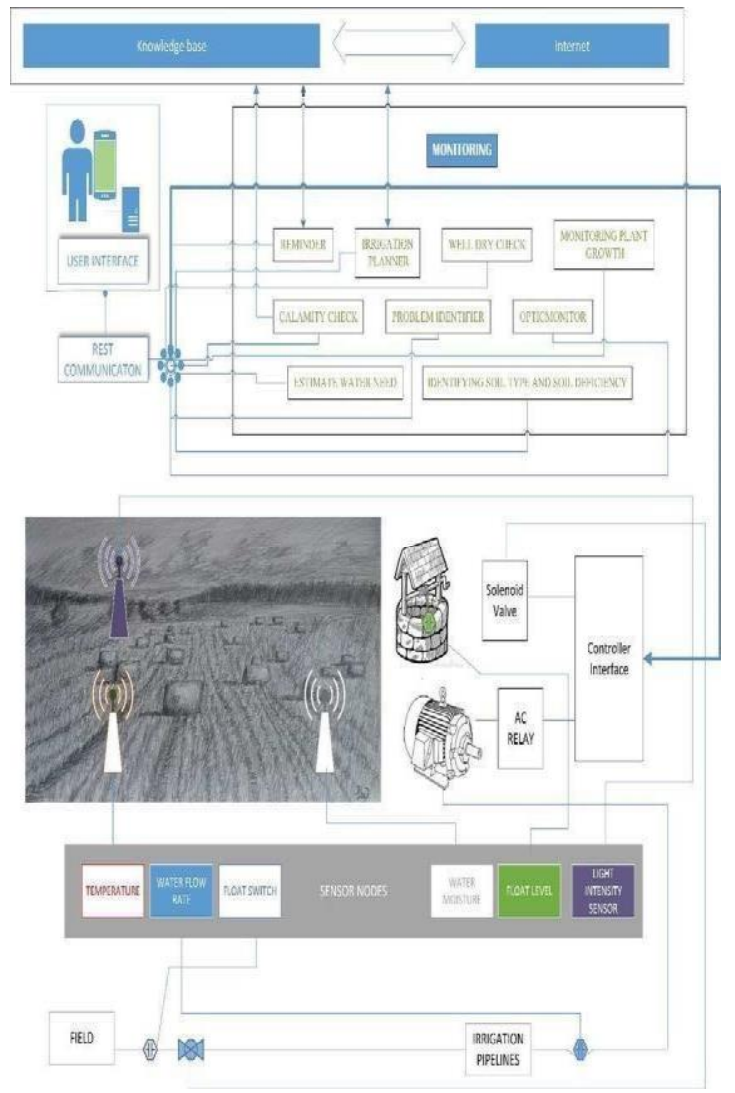

The project "Embedded LINUX based Agricultural Field monitoring and automation using IOT" was designed to develop an intelligent agricultural fields monitoring and controlling system using a Raspberry pi. The proposed system continuously monitors and controls different parameters like soil moisture, temperature and humidity, water, Light level. Alerting of the parameters is on LCD display and controlling can be done using electromagnetic relays.

\section{Future Scope:}

Integrating features of all the hardware components used have been developed in it. Presence of every module has been reasoned out and placed carefully, thus contributing to architecture of e-Agriculture Monitoring module the best working of the unit.

Secondly, using highly advanced IC's with the help of growing technology, the project has been successfully implemented. Thus, the project has been successfully designed and tested.

\section{Conclusion}

Integrating features of all the hardware components used have been developed in it. Presence of every module has been reasoned out and placed carefully, thus contributing to the best working of the unit. Secondly, using highly advanced IC's with the help of growing technology, the project has been successfully implemented. Thus, the project has been successfully designed and tested.

\section{References}

[1] G. V. Satyanarayana, SD.Mazaruddin, "Wireless Sensor Based Remote Monitoring System for Agriculture Using ZigBee and GPS", (C) 2013. The authors - Published by Atlantis Press.

[2] ALEXANDROS ZOGRAFOS, "Wireless Sensor-based Agricultural Monitoring System", Degree project in Communication Systems Second level, 30.0 HEC Stockholm, Sweden.

[3] V. Vinoth Kumar, R.Ramasamy, S.Janarthanan, M. VasimBabu, "IMPLEMENTATION OF IOT IN SMART IRRIGATION SYSTEM USING ARDUINO PROCESSOR ", International Journal of Civil Engineering and Technology (IJCIET) Volume 8, Issue 10, October 2017, pp. 1304-1314, Article ID: IJCIET_08_10_133.

[4] Shweta Bopshetty, Mrunali Yadav, Rithvika Rai, Sheril Silvister, Prof. Parth Sagar, "Monitoring and Controlling of Drip Irrigation using IOT with Embedded Linux Board", International Journal of Advanced Research in Computer and Communication Engineering Vol. 6, Issue 4, April 2017.

[5] Imtinungla, Samar Jyoti Saikia, Hemashree Bordoloi, "Embedded System based Agricultural Field Monitoring System", International Journal of Electronics Engineering Research.ISSN 0975-6450 Volume 9, Number 7 (2017) pp. 977-984.

[6] Dr. D.K. Sreekantha, Kavya.A.M, "Agricultural Crop Monitoring", 2017 11th International Conference on Intelligent Systems and Control (ISCO) 978-1-5090-27170/17/\$31.00 @2017 IEEE.

[7] M Manoj Kumar, P Rambabu, U Srinadh, T Satish, "Smart Agriculture-Using IoT in Field monitoring and Automation for Soil Moisture using Naive Bayes Prediction", International Journal of Advanced Information Science and Tech- 
nology (IJAIST) ISSN: 2319:2682 Vol.6, No.3, March 2017 DOI:10.15693/ijaist/2017.v6i3.20-25.

[8] A V L N Sujith, K Chandra Sekhar, "Automated Agriculture as a Service Using IoT", International Journal of Advanced Research in Computer Science and Software Engineering, Volume 7, Issue 5, May 2017.

[9] Pandurang H. Tarange, Rajan G. Mevekari, Prashant A. Shinde, "Web based Automatic Irrigation System using wireless sensor network and Embedded Linux board", 2015 International Conference on Circuit, Power and Computing Technologies [ICCPCT] 978-1-4799-70759/15/\$31.00 @2015 IEEE 